\title{
Test of the Equivalence Principle using Atomic Vacuum Energy Shifts
}

\author{
C. Alvarez" and R.B. Mann ${ }^{2}$ \\ Dept. of Physics, University of Waterloo Waterloo, ONT N2L 3G1, Canada
}

PACS numbers: $31.30 . J v, 14.60 . \mathrm{Cd}, 04.80 .+\mathrm{z}$

December 9, 2017

\begin{abstract}
We consider possible tests of the Einstein Equivalence Principle for quantum-mechanical vacuum energies by evaluating the Lamb shift transition in a class of non-metric theories of gravity described by the TH $\epsilon \mu$ formalism. We compute to lowest order the associated red shift and time dilation parameters, and discuss how (high-precision) measurements of these quantities could provide new information on the validity of the equivalence principle.
\end{abstract}

1email: calvarez@avatar.uwaterloo.ca
2 email: mann@avatar.uwaterloo.ca 
The Einstein Equivalence Principle (EEP) is foundational to our understanding of gravity. It ensures that a unique operational geometry can be attributed to spacetime, thereby guaranteeing that the effects of gravity on matter are accounted for in a purely geometric fashion. Metric theories, such as general relativity and Brans-Dicke Theory realize this principle by endowing spacetime with a symmetric, secondrank tensor field $g_{\mu \nu}$ that couples universally to all non-gravitational fields [1]. Non-metric theories do not have this feature: they violate universality by coupling auxiliary gravitational fields directly to matter, thereby permitting observers performing local experiments to detect effects due to their position and/or velocity in an external gravitational environment.

Empirical limits on such effects are set by gravitational red-shift and atomic physics experiments [2, 3], each of which compares relative frequencies of transitions between particular energy levels that are sensitive to any possible position or velocity dependence respectively. Significantly improved levels of precision for such experiments are anticipated in the next generation of gravitational experiments; for example, gravitational red shift experiments could be improved to one part in $10^{9}$ by placing a hydrogen maser clock on board Solar Probe, a proposed spacecraft (see ref. (4] and references therein). The dominant form of energy governing the transitions these experiments probe is nuclear electrostatic energy, although violations of the EEP due to other forms of energy (virtually all of which are associated with baryonic matter) have also been estimated [5]. Potential violations of the EEP due to effects such as vacuum energy shifts, which are peculiarly quantum-mechanical in origin (i.e. are due solely to radiative corrections) are not as well understood. In this paper we report on the results of an investigation into the effects that EEPviolating couplings have on Lamb-shift transition energies in Hydrogenic atoms. Details will appear in a forthcoming paper [6]. A test of the EEP for this form of energy provides us with a qualitatively new empirical window of the foundations of gravitational theory.

We begin with the action appropriate for Quantum Electrodynamics in a background gravitational field:

$$
S=\int d^{4} x \sqrt{-g}\left[\bar{\psi}(i \not \nabla+e \not A-m) \psi-\frac{1}{4} F_{\mu \nu} F^{\mu \nu}\right]
$$

where $\nabla$ is the covariant derivative, $F_{\mu \nu} \equiv A_{\nu, \mu}-A_{\mu, \nu}$ and $\not A=e_{\mu}^{a} \gamma_{a} A^{\mu}, e_{\mu}^{a}$ being the tetrad associated with the metric. Using the $T H \epsilon \mu$ formalism [7] (which encompasses a wide class of non-metric the- ories in addition to all metric theories) the general form of the static, spherically symmetric metric is $g_{\mu \nu}=\operatorname{diag}(-T, H, H, H)$ and $F_{\mu \nu} F^{\mu \nu}=2\left(\epsilon E^{2}-\right.$ $B^{2} / \mu$ ), where $\vec{E} \equiv-\vec{\nabla} A_{0}-\partial \vec{A} / \partial t$ and $\vec{B} \equiv \vec{\nabla} \times \vec{A}$. $\epsilon$ and $\mu$ are arbitrary functions of the Newtonian background potential $U=G M / r$ (which approaches unity as $U \rightarrow 0$ ) as are $T$ and $H$, which in general will depend upon the species of particles within the system (electrons in the present case).

Consider an atom that moves with velocity $\vec{u}$ relative to the preferred frame. The spacetime scale of atomic systems permits us to ignore spatial variations of $T, H, \epsilon$ and $\mu$; using this and a Lorentz transformation to transform fields and coordinates from the preferred frame to the rest frame of the atom (i.e. the moving frame), it may be shown that (1D) to $O\left(\vec{u}^{2}\right)$ reduces to [8]

$$
\begin{aligned}
S & =\int d^{4} x\left\{\bar{\psi}(i \not \partial+e \not A-m) \psi+J_{\mu} A^{\mu}\right. \\
& +\frac{1}{2}\left[E^{2}-B^{2}+\xi\left(\vec{u}^{2} E^{2}-(\vec{u} \cdot \vec{E})^{2}\right.\right. \\
& \left.\left.\left.+\left(1+\vec{u}^{2}\right) B^{2}-(\vec{u} \cdot \vec{B})^{2}+2 \vec{u} \cdot(\vec{E} \times \vec{B})\right)\right]\right\} .
\end{aligned}
$$

$J^{\mu}$ is the current associated with some external source (taken here to be a pointlike spinless nucleus).

The dimensionless parameter $\xi=1-H_{0} / T_{0} \epsilon_{0} \mu_{0} \equiv$ $1-c^{2}$ measures the degree of EEP violation, with "0" denoting the functions evaluated at the atomic system's center of mass and $c$ being the ratio of the limiting speed of electrons to the speed of light. The natural scale for $\xi$ is set by the magnitude of $U$, which empirically is much smaller than unity [4, permitting us to perform a perturbative analysis in $\xi$ and $\vec{u}$ of the radiative corrections associated with the action (2).

Perturbatively solving the field equations associated with (2) for the $A_{\mu}$ produced by a pointlike nucleus of charge $Z e$ at rest in the moving frame yields [8]

$$
\begin{aligned}
A_{0} & =\left[1-\frac{\xi}{2}\left(\vec{u}^{2}+(\vec{u} \cdot \hat{n})^{2}\right)\right] \phi \equiv \phi+\xi \phi^{\prime} \\
\vec{A} & =\frac{\xi}{2}[\vec{u}+\hat{n}(\vec{u} \cdot \hat{n})] \phi \equiv \xi \vec{A}^{\prime}
\end{aligned}
$$

where $\hat{n}=\vec{x} /|\vec{x}|, \phi=Z e / 4 \pi|\vec{x}|$, and $\vec{\nabla} \cdot \vec{A}=0$. The Dirac Hamiltonian may then be written as

$$
H=H_{0}+\xi H^{\prime}, \quad H^{\prime}=-e \phi^{\prime}+e \vec{\alpha} \cdot \vec{A}^{\prime}
$$

where $H_{0}$ corresponds to the standard Hamiltonian (with Coulomb potential only), and the primed fields are defined in (3). Using first-order perturbation theory on the Dirac equation, $H|n\rangle=E_{n}|n\rangle$, we can 
solve for $|n\rangle=|n\rangle^{0}+\xi|n\rangle^{\prime}$ and $E_{n}=E_{n}^{0}+\xi E_{n}^{\prime}$, which yields

$$
E_{2 S_{1 / 2}}-E_{2 P_{1 / 2}}=\xi \frac{u^{2}}{6} m(Z \alpha)^{4}+O\left((Z \alpha)^{6}\right)
$$

and so the $2 S_{1 / 2}-2 P_{1 / 2}$ degeneracy is lifted before radiative corrections are introduced. This nonmetric contribution to the Lamb shift is isotropic in the 3velocity $\vec{u}$ of the moving frame and vanishes when $\vec{u}=0$.

To lowest order in QED there are two types of radiative corrections to the energy levels of an electron bound in an external electromagnetic potential (shown in Fig. 1): the vacuum polarization (П) and self-energy $(\Sigma)$, along with a counterterm $(\delta C)$ that subtracts the analogous processes for a free electron. The radiative contributions to a state $|n\rangle$ will therefore be $\delta E_{n}=\langle n|\Sigma-\delta C+\Pi| n\rangle$. (a)

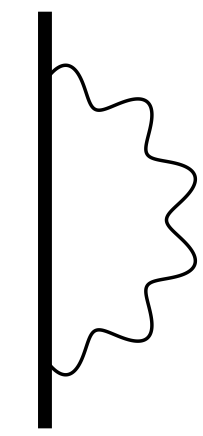

(b)

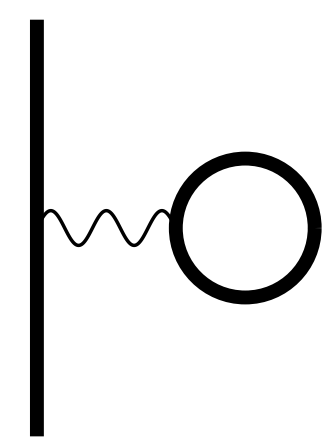

The Lamb shift calculation involves dealing with bound electron propagators, which in turn requires an approach adequate to sort out the external electromagnetic field dependence. We follow the method given by ref. [9] in which the bound propagator is separated into a term where the external potential acts only once, and another term where it acts at least twice. After a lengthy calculation similar to that described in ref. 9 we find to the required accuracy $O(\xi) O\left(\vec{u}^{2}\right) O\left(\alpha(Z \alpha)^{4}\right)$,

$$
\delta E_{n}=\frac{\alpha}{3 \pi m^{2}}\left[\left(1+\xi\left(\frac{3}{2}+\vec{u}^{2}\right)\right) \hat{C}+\xi u_{i} u_{j} \hat{C}^{i j}+\langle n|\hat{E}| n\rangle\right]
$$

with

$$
C^{i j}=2 \sum_{r}\left\langle r\left|p_{i}\right| n\right\rangle\left\langle n\left|p_{j}\right| r\right\rangle\left(E_{r}-E_{n}\right) \ln \left|\frac{E_{*}}{E_{n}-E_{r}}\right|
$$

$\hat{C} \equiv \hat{C}^{i i}$ and

$$
\begin{aligned}
& \hat{E}=4 \pi Z \alpha \delta(\vec{x})\left[\frac{19}{30}+\ln \left(\frac{m}{2 E_{*}}\right)\right. \\
& \left.+\quad \xi\left[-\frac{1}{30}-\frac{58}{45} \vec{u}^{2}+\left(\frac{3}{2}+\frac{2}{3} \vec{u}^{2}\right) \ln \left(\frac{m}{2 E_{*}}\right)\right]\right] \\
& +\quad 3 \frac{Z \alpha}{r^{3}}\left[\frac{1}{4}+\xi\left[\frac{1}{8}-\frac{\vec{u}^{2}}{2}-(\vec{u} \cdot \hat{n})^{2}\right]\right] \vec{\sigma} \cdot \vec{L} \\
& -\quad \xi \frac{Z \alpha}{r^{3}}\left[3(\vec{u} \cdot \hat{n})^{2}-\vec{u}^{2}\right]\left[\frac{14}{15}+2 \ln \left(\frac{m}{2 E_{*}}\right)\right] \\
& +\frac{\xi}{2} \frac{Z \alpha}{r^{2}}\left[\frac{7}{2} \vec{u} \cdot \hat{n} \vec{\sigma} \cdot(\vec{u} \times \vec{p})-\vec{\sigma} \cdot(\vec{u} \times \hat{n}) \vec{u} \cdot \vec{p}\right]
\end{aligned}
$$

where $E_{*}$ is a reference energy to be defined. We have omitted operators with odd parity (such as $\vec{u} \times \hat{n} \cdot \vec{\sigma}$ ) in (9), since their expectation values vanish for states of definite parity.

There is still an implicit dependence on $\xi$ and $\vec{u}$ coming from the Dirac states (due to the non coulombic behavior of the electromagnetic source (3i)), which modifies the answer in (7) to

$$
G_{\mu \nu}=-(1+\xi) \frac{\eta_{\mu \nu}}{k^{2}}+\xi \frac{\gamma^{2}}{k^{2}}\left[\eta_{\mu \nu} \frac{(\beta \cdot k)^{2}}{k^{2}}+\beta_{\mu} \beta_{\nu}\right]
$$

for the photon propagator, where $\eta_{\mu \nu}$ is the Minkowski tensor with signature $(+---), \beta^{\mu} \equiv$ $(1, \vec{u})$ and $\gamma^{2} \equiv\left(1-\vec{u}^{2}\right)^{-1}$.

As in the metric case, regularization and renormalization processes are needed. We choose the cutoff method to deal with the divergences, absorbing them by proper redefinitions of the parameters of the theory, which now include the $T H \epsilon \mu$ functions. Even though we reformulate quantum electrodynamics without the symmetries given by local position and local Lorentz invariance, the theory still remains gauge invariant and the self consistency of the Ward Identities is straightforwardly checked.

$$
\delta E_{n}=\frac{\alpha}{3 \pi m^{2}}\left[\left(1+\xi\left(\frac{3}{2}+\vec{u}^{2}\right)\right) \hat{C}^{0}+\xi u_{i} u_{j} \hat{E}^{i j}+^{0}\langle n|\hat{E}| n\rangle^{0}\right]
$$

with

$$
u_{i} u_{j} \hat{E}^{i j}=u_{i} u_{j} \hat{C}^{i j}+\hat{C}^{\prime}+\left({ }^{0}\left\langle n\left|\hat{E}_{\xi=0}\right| n\right\rangle^{\prime}+\text { h.c. }\right)
$$

where $\hat{C}^{\prime}$ groups all the terms in eq. (8) depending on the perturbative corrections $\left(|n\rangle^{\prime}\right)$ and $\left(E_{n}^{\prime}\right)$ to the states $|n\rangle$ and energies $\left(E_{n}\right)$. These are needed not only for the $|n\rangle$ state related to the level shift, but for all the intermediate states introduced by (B) as well.

If we now define the reference energy $\left(E_{*}\right)$ as in the metric case [10], and evaluate eq. (10) for the Lamb states, we can write the total contribution for, say, the $2 S_{1 / 2}-2 P_{1 / 2}$ Lamb shift (including the non radiative contribution (5)) as: 


$$
\begin{aligned}
& \Delta E_{L}=\frac{m}{6 \pi}(Z \alpha)^{4} \alpha\left\{\ln \frac{1}{\alpha^{2}}-2.084+\xi\left[\frac{3}{2} \ln \frac{1}{\alpha^{2}}-4.534\right.\right. \\
& \left.\left.+\vec{u}^{2}\left[\frac{\pi}{\alpha}-3.486+\frac{2}{3} \ln \frac{1}{\alpha^{2}}-0.011 \cos ^{2} \theta\right]+u_{i} u_{j} \Delta \hat{\epsilon}^{i j}\right]\right\}
\end{aligned}
$$

Here $\theta$ represents the angle between the atom's quantization axis and the frame velocity $\vec{u}$ and $\Delta \hat{\epsilon}^{i j} \equiv$ $2 \Delta \hat{E}^{i j} /\left((Z \alpha)^{4} m^{3}\right)$

Useful empirical information may be extracted by calculating the gravitational redshift and timedilation parameters associated with (12). In a redshift experiment the local energies at emission $w_{\text {em }}$ and at reception $w_{r e c}$ of a photon transmitted between observers at different points in an external gravitational field are compared in terms of $Z=$ $\frac{w_{e m}-w_{r e c}}{w_{e m}} \equiv \Delta U(1-\Xi)$, whereas experiments comparing atomic energy transitions between the moving $\left(w_{u}\right)$ and preferred $\left(w_{0}\right)$ frames may be described via $w_{u}=w_{0}\left(1-[A-1] \frac{\vec{u}^{2}}{2}\right)$. The anomalous redshift $(\Xi)$ and time dilation $(A)$ parameters may be computed from the anomalous passive and inertial mass tensors using standard techniques [4, 5]. After rescaling the action so that $\alpha \propto \sqrt{H / T} / \epsilon$, we find for the Lamb shift transition (12)

$$
\Xi^{L}=3.424 \Gamma_{0}-1.318 \Lambda_{0}
$$

and

$1-A^{L}=\frac{\xi}{7.757}\left[\frac{\pi}{\alpha}+3.074-0.011 \cos ^{2} \theta+\frac{u_{i} u_{j}}{\vec{u}^{2}} \Delta \hat{\epsilon}^{i j}\right]$

with

$$
\left.\left.\Gamma_{0} \equiv \frac{T_{0}}{T_{0}^{\prime}} \ln \left[\frac{T \epsilon^{2}}{H}\right]^{\prime}\right|_{0} \quad \Lambda_{0} \equiv \frac{T_{0}}{T_{0}^{\prime}} \ln \left[\frac{T \mu^{2}}{H}\right]^{\prime}\right|_{0}
$$

We emphasize that qualitatively new information on the validity of the EEP will be obtained by setting new empirical bounds on the parameters $\xi, A_{L}$ and $\Xi_{L}$ which are associated with purely leptonic matter. Comparatively little is known about empirical limits on EEP-violation in this sector [11]. Previous experiments have set the limits $[3]\left|\xi_{B}\right| \equiv$ $\left|1-c_{B}^{2}\right|<6 \times 10^{-21}$ where $c_{B}$ is the ratio of the limiting speed of baryonic matter to the speed of light, and [2] $\left|3 \Gamma_{0}-\Lambda_{0}+2 \Delta\right|<2 \times 10^{-4}$ and $\Gamma_{0}-\Gamma_{B 0} \equiv \Delta \equiv \Lambda_{0}-\Lambda_{B 0}$ where $\Gamma_{B 0}$ and $\Lambda_{B 0}$ are quantities analogous to those in eq. 115) for baryons, where for simplicity we have assumed $T_{B 0}=T_{0}$. This latter experiment involves interactions between nuclei and electrons and so does not (at least to the leading order to which we work) probe the leptonic sector in the manner that Lamb-shift experiments would.
The coefficient $A_{L}$ depends upon $\Delta \hat{\epsilon}^{i j}$, the evaluation of which involves the numerical computation of the sum in (11). The contribution in eq. (14) from the Dirac part of the energy (proportional to $\frac{1}{\alpha}$ ), produces an overall shift only. Assuming that EEPviolating contributions to $\Delta E_{L}$ are bounded by the current level of precision for the Lamb shift [12], and that $|\vec{u}|<10^{-3}[4]$, we find the dominant contribution to (14) is due to the purely radiative part of (12), yielding the bound $|\xi|<10^{-5}$. This limit is comparable to that noted in a different context by Greene et. al. [13. We note that anisotropic effects in (14) arise solely from radiative corrections.

Improvement on such bounds will be a challenge to experimentalists because of the intrinsic uncertainties of excited states of Hydrogenic atoms. Setting empirical bounds on $\Xi_{L}$ will involve measuring the frequency shifts of an atomic clock based on the Lamb shift transition, either by comparing two such transitions at different points in a gravitational potential or by performing a 'clock-comparison' type of experiment between a 'Lamb-shift clock' and some other atomic frequency standard [4]. The former experiment would appear unfeasible since the anticipated redshift in the background potential of the earth $\left(\approx 10^{-9}\right)$ is much smaller than any foreseeable improvement in the precision of Lamb-shift transition measurements [12]. One would at least need to perform the experiment in a stronger gravitational field (such as on a satellite in close solar orbit) with 1-2 orders-of-magnitude improvement in precision. The latter measurement is, in principle, sensitive to the absolute value of the local potential [11, 14], whose magnitude has recently been estimated to be $\approx 10^{-5}$ due to the local supercluster [15. The best hope would appear to be in exploiting anticipated improvements in precision [12 to obtain better bounds on $\xi$ via (12).

Finally, we note that our calculation has assumed that positrons and electrons have equivalent couplings to the gravitational field. If we drop this assumption [16, we find that there is an additional contribution to (12) due to $\xi_{e^{+}} \neq \xi_{e^{-}}$. This contribution is due entirely to radiative corrections. Making the same comparisons as above, we find the most stringent bound on this quantity to be $\left|\xi_{e^{+}}\right|<10^{-3}$ from present experiments. In a similar vein, one could also consider tests of the EEP for muonic atoms to obtain bounds on $\xi_{\mu}$. We shall report on this elsewhere [6].

The intrinsically quantum-mechanical character of the radiative corrections will, we hope, motivate the development of new EEP experiments based on the Lamb shift transition, thereby extending our understanding of the validity of the equivalence princi- 
ple into the regime of quantum-field theory.

\section{Acknowledgments}

This work was supported in part by the Natural Sciences and Engineering Research Council of Canada. We are grateful to C.M. Will for his initial encouragement in this work and to E. Hessels for discussions.

\section{References}

[1] K.S. Thorne, A. P. Lightman and D. L. Lee, Phys. Rev. D 7, 3563 (1973).

[2] R. F. C. Vessot and M.W. Levine, Gen. Relativ. Gravit. 10, 181 (1979).

[3] J. D. Prestage et al., Phys. Rev. Lett. 54, 2387 (1985); S. K. Lamoreaux et al., ibid. 57, 3125 (1986); T. E. Chupp et al., ibid. 63, 1541 (1989).

[4] C. M. Will, Theory and Experiment in Gravitational Physics, 2nd edition (Cambridge University Press, Cambridge, 1992).

[5] M. P. Haugan and C. M. Will, Phys. Rev. Lett. 37, 1 (1976); Phys. Rev D. 15, 2711 (1977)

[6] C. Alvarez and R.B. Mann, "Test of the Equivalence Principle by Lamb Shift Energies" WATPHYS-TH95/02.

[7] A. P. Lightman and D. L. Lee, Phys. Rev. D 8, 364 (1973).

[8] M. D. Gabriel and M. P. Haugan, Phys. Rev. D 41, 2943 (1990).

[9] M. Baranger, H. A. Bethe, and R. P. Feynman, Phys. Rev. 92, 482 (1953)

[10] C. Itzykson and J. B Zuber, Quantum Field Theory (McGraw-Hill, International Editions, 1987).

[11] R.J. Hughes, Contemporary Physics 34177 (1993).

[12] M.I. Eides and V.A. Shelyuto, Phys. Rev. A52 954 (1995).

[13] G. Greene et. al., Phys. Rev. D44 2216 (1991).

[14] M.L. Good, Phys. Rev. 121311 (1961).

[15] I.R. Kenyon, Phys. Lett. B237 274 (1990).

[16] L. Schiff, Proc. Nat. Acad. Sci. 4569 (1959). 